\title{
A New Method to Enhance the Clustering Algorithm
}

\author{
Abolfazl Zanghaei, Mohammadjavad Abolhassani, Alireza Ahmadian, Mohammad Reza Ay, and \\ Houshang Saberi
}

\begin{abstract}
In this paper we have presented a new method to enhance the clustering algorithm. Clustering is a method to label points in a two or more dimensional scatter plot. Some algorithms such as FCM, KNN or K-means implement this, but the problem occurs in the case that the clusters are close to each other or even they have overlaps (like neural data which we first encountered). In this state the human observer can detect some clusters on the scatter plot but the algorithms fail or they offer different clusters. To solve this problem we propose that the TPS transform on the primary data (clusters) can improve the clustering solution which is more likely to human perception of the clusters. To show the validity of our method we have used the SPSS analyses.
\end{abstract}

Index Terms-FCM, TPS, subtractive clustrering, image registration.

\section{INTRODUCTION}

In many cases we extract features from a signal, image. These features may be amplitude, frequency, special Fourier coefficients, PCA components, or etc. Instead of processing the source signal or raw data we can process these features. For example to cluster neural spikes, we can first calculate the principle components (PCA) of signal. These features are points in a scatter plot. Clustering of these features is easier than the neural spikes [1], [2].

As we were working on neural data, we found that when our signal is transformed on a feature vector (scatter plot) usually features were close to each other and even they had overlaps. In other words in the scatter plot we saw some clusters which have overlaps. Figure 1 shows an example of these overlaps of clusters. However, in figure 2 three different clusters without any overlaps can be detected.

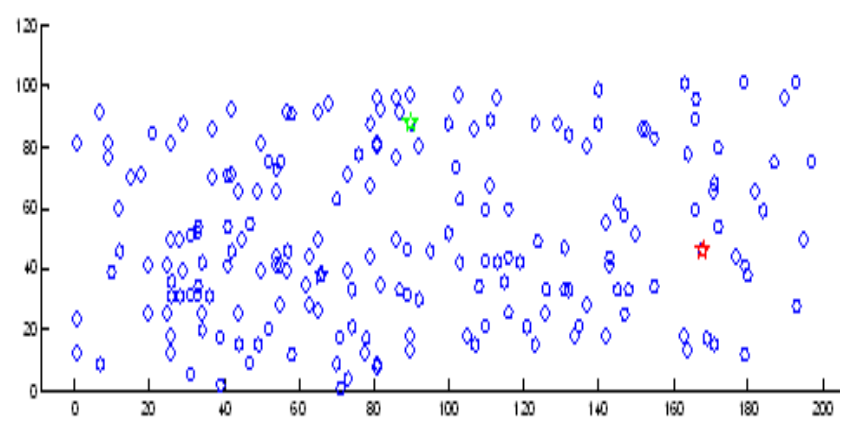

Fig. 1. Overlapping clusters

Manuscript received October 20, 2012; revised November 27, 2012.

Abolfazl Zanghaei, Mohammadjavad Abolhassani, Alireza Ahmadian, and Mohammad Reza Ay are with Research Centre for Science \& Technology in Medicine, Department of biomedical Eng \& biophysics, Tehran University of Medical Sciences, Tehran, Iran (e-mail: zanghaei@razi.tums.ac.ir)

Houshang Saberi is with Brain \& Spinal Injury Repair Research Center Tehran University of Medical Sciences, Tehran, Iran.

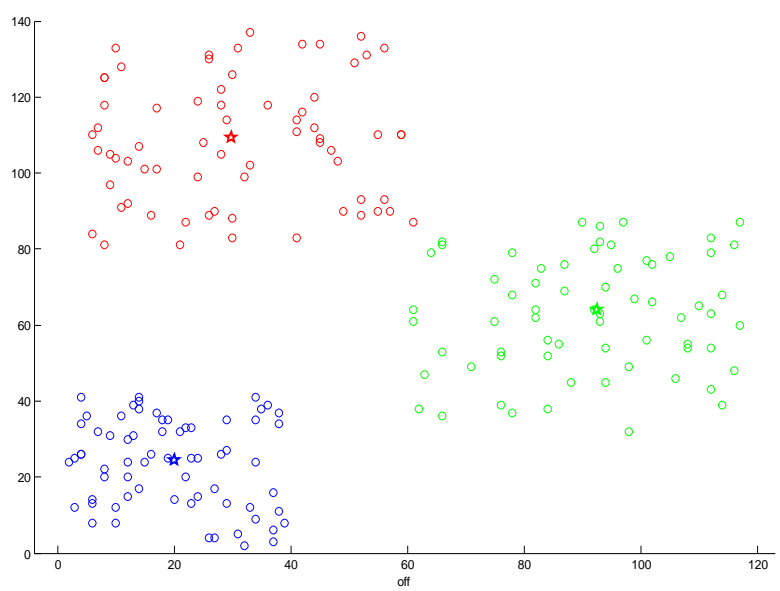

Fig. 2. Three different clusters

Applying any clustering algorithm on figure 2 usually will have unique answer. However, in figure 1 it is hard for the clustering algorithm to detect the clusters exactly or even with a very small error.

The data points on figure 1 should be transformed such that the clustering result resembles the human perception of the clusters. To achieve this, first some centers in the data points should be determined. These centers show the probable locations of clusters. It can be shown that subtractive clustering can do this job.

\section{Subtractive Clustering}

If there is not a clear idea how many clusters are present in a given set of data, subtractive clustering, is a fast, onepass algorithm for estimating the number of clusters and the cluster centers in a set of data points. The subtractive clustering method assumes each data point is a potential cluster center and calculates a measure of the likelihood that each data point would define the cluster center, based on the density of the surrounding data points. The algorithm selects the data point with the highest potential to be the first cluster center, then removes all the data points in the vicinity of the first cluster center (as it is determined by radius), in order to determine the next data cluster and its center location, this process is iterated until all of the data points are within the radius of a cluster center [3]. The subtractive clustering provides a powerful comparison of clusters and is a first step for finding non-obvious (hidden) differences and minimizing the human prejudice during the analysis [4].

In this paper first we have a global estimation of probable clusters using this method. After finding the initial guess of centers all the data should be transformed by applying the TPS transform. 


\section{TPS TRANSFORM}

Thin Plate Spline (TPS) is a method to warp an image in accordance with input and base control points. In other words it is a method for image registration. Given a set of data points, a weighted combination of thin plate spline centered about each data point gives the interpolation function that exactly passes through the points while minimizing the so-called bending energy. The name "Thin Plate Spline" refers to a physical analogy involving the bending of a thin sheet of metal. There are many references for this transformation [3]-[7].

Generally speaking the TPS acts as a function that transforms all the points of a grid to another warped grid as shown in equation (1).

$$
T(x, y, z)=\left(\begin{array}{c}
x^{\prime} \\
y_{\cdot} \\
z^{\prime} \\
1
\end{array}\right)=\left(\begin{array}{cccccc}
a_{00} & a_{01} & . . & . . & . . & a_{0 n} \\
a_{10} & . . & . . & . . & . . & a_{1 n} \\
a_{20} & . . & . . & . . & . . & a_{2 n} \\
0 & 0 & . . & . . & . . & 1
\end{array}\right)\left(\begin{array}{c}
\theta_{1}(x, y, z) \\
\theta_{2}(x, y, z) \\
. . \\
. . \\
\theta_{n}(x, y, z) \\
1
\end{array}\right)
$$

In the equation (1) $x, y, z$ are the input points and $x^{\prime}, y^{\prime}, z^{\prime}$ are warped points. $a_{i j} \mathrm{~s}$ are the coefficients that are determined by the control points. Equation (2) shows a general form for a TPS transfer function.

$$
T(x)=P(x)+\sum_{i=1}^{n} A \cdot g\left(r_{i}\right)
$$

In this equation $P(x)$ is a polynomial that determines the rigid or affine function and $g\left(r_{i}\right)$ is a Radial Basis Function and $r_{i}$ is Euclidean distance of control points. $A$ is a matrix which is related to the control points. The control points determine the pattern of movement of certain points and the deformation of the grid.
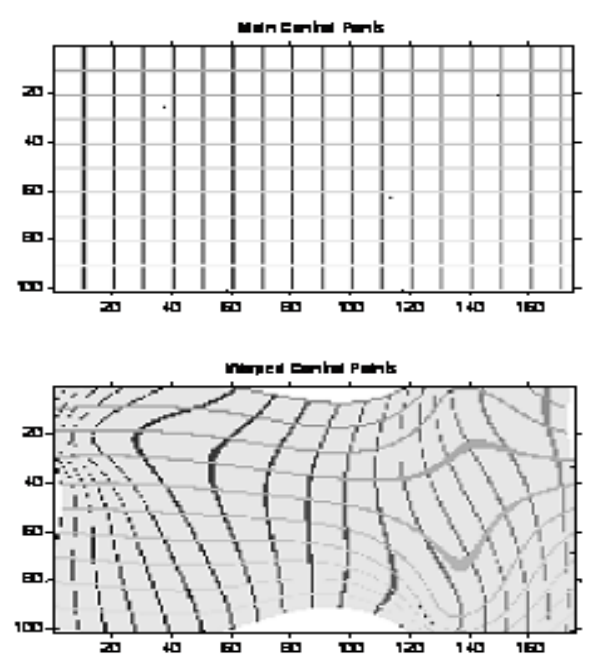

Fig. 3. Deformation of a grid which tries to move all the points, closer to the primary centers.

How this transform can be used in clustering? As it is shown in the previous section, the centers of the clusters can be found with the subtractive clustering method. If we try to transform the data (like the scatter plot in figure 1) in such a way, that all the points move to the primary clusters (which is defined by the subtractive clustering), then we have achieved a novel method that helps the algorithm for a better clustering. In order to distinguish the overlapping points within two clusters, the overlapping points must be moved more than others, in other words a non linear transform or non rigid transform must be used as shown in figure 3.

However, first the control points must be determined.

\section{SElection OF CONTROL PoInts}

First the center of the primary centers is calculated, then lines are drawn from this point to all the other centers. After this, the control points are selected using these two methods:

1) The control points are selected such that they move from the 1.2 and 0.8 times of the radius (figure 4).

2) The control points are selected such that they move from half of the angle (bisector) with the adjacent center to the desired center (figure 5).

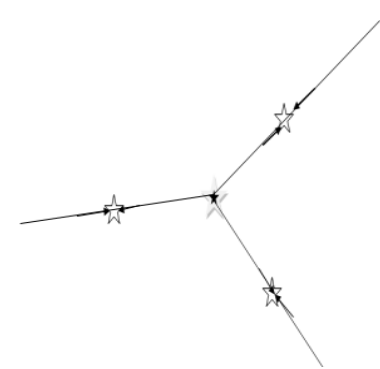

Fig. 4. Movement of control points from .8 and 1.1 to centers

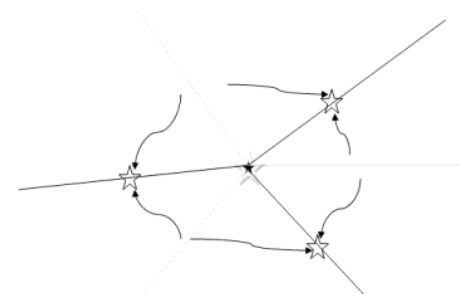

Fig. 5. Movement of control points from bisector to the primary centers.

To assure these control points and TPS transform do not distort the data, after the selection of the control points, cluster validation criteria must be met.

\section{Cluster VALIDATION}

Cluster validation shows how much between class distance is large, and how much within class distance is small. In the next section we show that our method has improved these factors. For the assessment of the validity of our method a random scatter plot is generated, where the points are labeled in this plot. Then the points are clustered by the FCM method, and finally the error labels are counted. For the comparison we also first transform the random plot scatters and then cluster the scatter plot with the FCM method. This time, misclassification errors will also be counted.

\section{RESULTS}

We generate the random scatter plot 100 times then we measured the mean of errors, between and within class distances. In order to asses our method the results of Kappa 
measurements without our method (clustering with FCM) and with the new method are presented in table I and table II. These tables are the result of data analysis by SPSS 16.0 software.

TABLE I: KAPPA MEASURMENT, BEFOR AND AFTER APPLying THE NEW METHOD

\begin{tabular}{|c|c|c|c|c|}
\hline Kappa & Value & $\begin{array}{c}\text { Asymp. Std. } \\
\text { Error }\end{array}$ & Approx. T & $\begin{array}{c}\text { Approx. } \\
\text { Sig. }\end{array}$ \\
\hline $\begin{array}{c}\text { Kappa before our } \\
\text { method }\end{array}$ & 0.87 & 0.003 & 150.767 & 0 \\
\hline $\begin{array}{c}\text { Kappa after our } \\
\text { method }\end{array}$ & 0.891 & 0.003 & 154.369 & 0 \\
\hline
\end{tabular}

Although some distances are larger than before applying the new method but paired samples test shows they are generally smaller with a good P-Value. As it shown in table II the clusters are more compact.

TABLE II: PARED SAMPLE TEST WITH SPSS

\begin{tabular}{|c|c|c|c|c|}
\hline Test & Mean & $\boldsymbol{N}$ & $\begin{array}{c}\text { Std. } \\
\text { Deviation }\end{array}$ & $\begin{array}{c}\text { Std. } \text { Error } \\
\text { Mean }\end{array}$ \\
\hline $\begin{array}{c}\text { After } \\
\text { Method }\end{array}$ & 70.5484 & 100 & 27.4504 & 2.7450 \\
\hline $\begin{array}{c}\text { Before } \\
\text { Method }\end{array}$ & 87.4166 & 100 & 28.6353 & 2.8635 \\
\hline
\end{tabular}

Our results show that tests are normal and misclassification error is reduced at least by half i.e. without the method the mean of errors is 6 and after the method is 3 . For 100 times test the Kappa factor (measurement of agreement) improves from 0.87 to 0.891 which shows the reduction of error.

\section{A ReAl APPLICATION}

This method can be used in online clustering of the neural spikes. For this application we first extract the features of neural spikes (scatter plot) and then transform data points with the TPS, and finally cluster with the FCM method.

\section{CONCLUSION}

In this paper we presented a new method to improve the clustering algorithm (FCM) and we showed that our method improves the clustering method with a TPS transformation on the input data points. It also helps the FCM to works well and it reduces the different clustering by FCM

\section{ACKNOWLEDGEMENT}

This work is supported by Medical Physics \& Biomedical Engineering Department of Tehran University of Medical Science (tums.ac.ir) and Research Center for the Science and Technology in Medicine (www. rcstim.tums.ac.ir).

\section{REFERENCES}

[1] M. S. Lewickiy, "A review of methods for spike sorting: the detection and classification of neural action potentials," Network: Comput, Neural Syst. 9, R53-R78, 1998.

[2] P. M. Zhang, J. Y. Wu, Y. Zhoub, P. J. Liang, and J. Q. Yuan, "Spike sorting based on automatic template reconstruction with a partial solution to the overlapping problem," Elsevier, Journal of Neuroscience Methods, vol. 135, pp. 55-65, 2004.

[3] M. Alata, M. Molhim, and A. Ramini, "Optimizing of Fuzzy CMeans Clustering Algorithm Using GA." World Academy of Science, Engineering and Technology, vol. 39, 2008

[4] N. S. Jacob, R. Lisa, S. Peter, C. L. Rosemary, and F. L. James, "Subtractive clustering analysis: a novel data mining method for finding cell subpopulations," Imaging, Manipulation, and Analysis of Biomolecules and Cells: Fundamentals and Applications III. Ramesh, in Proceedings of the SPIE, vol. 5699, pp. 354-361 2005.

[5] J. Kim, "Intensity based Image Registration using Robust Similarity Measure and Constrained Optimization: Applications for Radiation Therapy," A dissertation submitted in partial ful_llment of the requirements for the degree of Doctor of Philosophy, University of Michigan 2004, Chapter 4.

[6] B. Zitová and J. Flusser, "Image registration methods: a survey," Image Vision Comput, vol. 21, no. 11, pp. 977-1000, 2003.

[7] M. A. Wirth, J. Narhan, and D. Gray, "Non-rigid mammogram registration using mutual information," SPIE Medical Imaging: Image Processing, San Diego USA, February 2002, vol. 4684, pp. 562-573.

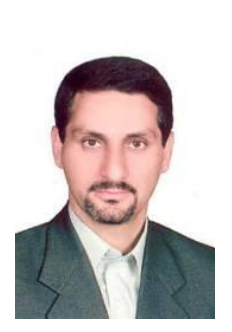

Mohammadjavad Abolhassani was born in Tehran in 1965. He was award a Ph.D. in biomedical systems from Imperial College London of UK in 1994. He is associate professor of medical physics and biomedical engineering department of Tehran University of Medical Sciences and also head of biomedical group of Research Center for Science \& Technology in Medicine, $\mathrm{He}$ has published many papers in national and international journals. He published two books, Ultrasound Physics \& Instrumentation in 2007 (Translated in Persian) and Medical Physics for Medical \& Dentistry Students in 2006 (Persian). His resaerch interest includes; Medical Instrumentation, Medical Ultrasound Instrumentation, Ultrasound Image Processing, Otoacoustic Emission Systems, and Biological Signal Processing. Dr. Abolhassani is member of IEEE ( Institute of Electrical and Electronics Engineers), ISBME (Iranian Society of Biomedical Engineers), IAMP (Iranian Association of Medical Physics), IOMP ( International Organization of Medical Physics), and Iranian Examination \& Evaluation Board of Biomedical Engineering also he has two scientific awards; Innovation prize of International Kharazmi Festival 2003, and Innovation prize of Medical Razi festival 2005.

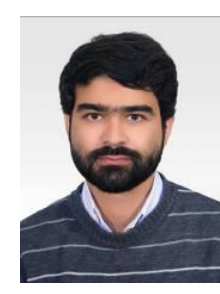

Abolfazl Zanghaei was born in Neyshaboor, Iran in 1982. He received his Msc degree in biomedical Engineering (bioelectrics) from Tehran University of medical sciences in 2010. He has lectured some courses in Azad University and also researcher of biomedical group of Research Center for Science \& Technology in Medicine (RCSTIM). His resaerch interest is Biomedical Engineering. Eng Zanghaei is member of ISBME (Iranian Society of Biomedical Engineers), IRCEO (Iranian Construction Engineering Organization)

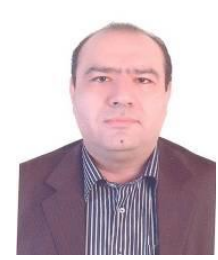

Hooshang Saberi was born in Tehran in $1964 \mathrm{He}$ was awarded a specialty of neurosurgery in 1995. He is Associate Professor of neurosurgery department of Tehran University of Medical Sciences. Deputy of Brain and Spinal Injuries Repair Research Center, Imam Khomeini Hospital. He has published many papers in national and international journals. Dr. Saberi is member of Iran association of Neurological Surgeon

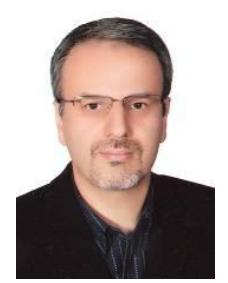

Alireza Ahmadian was born in Tehran in 1965. He received his $\mathrm{PhD}$ degree in department of biomedical engineering of Imperial College of Science, Technology in Medicine, London, 1997. He was nominated for a postdoctoral position at Kings College London, Multimedia Lab where he spent two years working on 3D medical image compression and transmission using wavelets transform. He is currently an Associate Professor at Tehran University of Medical Sciences, Biomedical Systems \& Biophysics Group. He also holds the deputy head of Research Centre for Science and Technology in Medicine, RCSTIM where most of advanced research projects in the field of biomedical engineering are ongoing in Iran. He also chairs Image Guided Surgery research group where most of innovative projects in the field of image guided navigation systems are carrying out. Dr. Ahmadn is also a senior member of IEEE. 